\title{
Free Energy Landscapes of Sodium lons Bound to DMPC-Cholesterol Membrane Surfaces at Infinite Dilution ${ }^{\dagger}$
}

\begin{abstract}
Jing Yang, ${ }^{a}$ Massimiliano Bonomi, ${ }^{b}$ Carles Calero, ${ }^{* c}$ and Jordi Martí, ${ }^{* a}$
Exploring the free energy landscapes of metal cations on phospholipid membrane surfaces is important for the understanding of chemical and biological processes in cellular environments. Using metadynamics simulations we have performed systematic free energy calculations of sodium cations bound to DMPC phospholipid membranes with cholesterol concentration varying between $0 \%$ (cholesterol-free) and 50\% (cholesterol-rich) at infinite dilution. The resulting free energy landscapes reveal the competition between binding of sodium to water and to lipid head groups. Moreover, the binding competitiveness of lipid head groups is diminished by cholesterol contents. As cholesterol concentration increases, the ionic affinity to membranes decreases. When cholesterol concentration is greater than $30 \%$, the ionic binding is significantly reduced, which coincides with the phase transition point of DMPC-cholesterol membranes from a liquid-disordered phase to a liquid-ordered phase. We have also evaluated the contributions of different lipid head groups to the binding free energy separately. The DMPC's carbonyl group is the most favorable binding site for sodium, followed by DMPC's phosphate group and then the hydroxyl group of cholesterol.
\end{abstract}

\section{Introduction}

The interactions between metal cations and phospholipid membranes have drawn great attention during last decades ${ }^{1,2}$. Several experiments revealed that metal cations are bound to the negative charged head groups of phospholipid membranes ${ }^{3-6}$. Meanwhile, numerous molecular dynamics (MD) simulations have been performed to study the binding of metal cations at phospholipid membranes from an atomic point of view ${ }^{6-13}$. However, most of the experiments and simulation works on ionic binding to phospholipid membranes have been devoted to cholesterol-free environments. Cholesterol is a crucial component in mammalian cell membranes, constituting up to $50 \%$ of their weight ${ }^{14}$. Cholesterol can modulate the structural and mechanical properties of membranes and can induce a phase transition from a liquid disordered phase to a liquid ordered phase ${ }^{15-17}$. Therefore, the study of the binding of metal cations to cholesterol-containing membranes is of interest to understand ionic binding in realistic cellular environments. Only recently, experiments by Iraolagoitia et al. showed that cholesterol significantly reduced the $\mathrm{Ca}^{2+}$ binding to

\footnotetext{
${ }^{a}$ Department of Physics and Nuclear Engineering, Technical University of CataloniaBarcelona Tech, B4-B5 Northern Campus, Jordi Girona 1-3, 08034 Barcelona, Catalonia, Spain; E-mail: jordi.marti@upc.edu

${ }^{b}$ Department of Chemistry, University of Cambridge, Lensfield Road, Cambridge CB2 1 EW, United Kingdom

c Center for Polymer Studies and Department of Physics, Boston University, 590 Commonwealth Avenue, Boston, Massachusetts 02215, United States; E-mail: ccalero@bu.edu

$\dagger$ Electronic Supplementary Information (ESI) available
}

membranes ${ }^{18}$. More recently, Magarkar et al. performed both experiments and simulations, revealing that increasing cholesterol concentration decreased $\mathrm{Na}^{+}$binding ${ }^{19}$.

The binding of metal cations at membranes in aqueous solution can result in several possible bound configurations, as a consequence of the loss of water molecules and the gain of lipid atoms in the ion's first hydration shell. Exploring the binding processes and bound states of metal cations at phospholipid membrane surfaces is important for the understanding of chemical and biological processes such as binding, hydration, leakage, and dissociation in cellular environments. Nevertheless, a comprehensive study of the relative stabilities of different bound states is a difficult experimental task, and also difficult for classical MD simulations because of the high free energy barriers among various bound states. To circumvent such difficulty, free energy calculations applying enhanced sampling techniques can be employed.

Recently, we have proposed a general methodology to explore the free energy landscapes for ions at biological interfaces and obtained the relative stabilities of different bound states for biologically relevant cations $\mathrm{Na}^{+}, \mathrm{K}^{+}, \mathrm{Ca}^{2+}$, and $\mathrm{Mg}^{2+}$ at cholesterolfree membranes ${ }^{20}$. In this work, we further apply this methodology to cholesterol-containing membranes and have performed systematic free energy calculations of $\mathrm{Na}^{+}$bound to phospholipid membranes of various cholesterol concentrations at infinite ion dilution. The resulting free energy landscapes further validate our methodology at membrane interfaces with higher complexity and unveil the cholesterol effects on $\mathrm{Na}^{+}$binding at phospholipid membranes. 



Fig. 1 Binding free energy difference $\Delta \mathrm{F}$ as a function of the coordination number of lipid oxygens (CLP) and the coordination number of water oxygens (CWT) for $\mathrm{Na}^{+}$bound to DMPC membranes with cholesterol concentrations [CHOL] varying between $0 \%$ and $50 \%$. The origin of free energy is given by the binding state with minimum free energy, with $C L P=0$ and $C W T=5$.

\section{Methods}

\subsection{System Setup and Equilibration.}

Six sets of simulations were performed to study the binding of $\mathrm{Na}^{+}$to neutral zwitterionic phospholipid membranes with different cholesterol content. CHARMM-GUI ${ }^{21,22}$ was employed to generate six sets of lipid bilayers with cholesterol concentrations [CHOL] varying between $0 \%$ and 50\%. Each system consisted of 72 cholesterol / dimyristoylphosphatidylcholine (DMPC) 23,24 molecules, 3600 water molecules described by the modified TIP3P model 25,26 , and a pair of $\mathrm{Na}^{+}$and $\mathrm{Cl}^{-}$described by the CHARMM36 force field ${ }^{27,28}$. In contrast to other force fields (such as GROMOS), the CHARMM36 force field generates a less pronounced adsorption of $\mathrm{Na}^{+}$ions on phosphatidylcholine (PC) membranes, which was shown to be consistent with structural Xray measurements and experiments on chain ordering of PC lipid bilayers in the presence of $\mathrm{NaCl}^{29}$. To achieve such agreement with experiment, in CHARMM36 the Lennard-Jones parameters characterizing the interaction of $\mathrm{Na}^{+}$with selected groups in the phospholipid are not calculated following the standard arithmetic combining rules, but they are determined specifically for each atom pair (NBFIX) ${ }^{30}$. The need for a specific description of cer- tain pair interactions shows the limitation of point charge force fields, which cannot account for charge transfer or polarization effects 31

The molar ratios of cholesterol/DMPC were set to 0/72 (0\%), 8/64 (11.1\%), 14/58 (19.4\%), 22/50 (30.6\%), 30/42 (41.7\%), and $36 / 36(50 \%)$. Due to the limited size of the system, our simulations can only capture the effect of short wave undulations on the adsorption of ions to the membrane. However, we believe that, being ion-binding presumably a local effect with characteristic length scales in the order of the nanometer, the relevant undulations are the short wavelength modes, which are well accounted for even by small systems ${ }^{32}$. We have investigated the effect of the limited size of the simulated system by comparing the occupancy distribution of the lower ion binding states obtained from unbiased simulations of systems containing 72 and 128 DMPC/cholesterol molecules with $0 \%, 20 \%$, and $30 \%$ of cholesterol (see Fig. S3 in the Supplementary Information). We obtain very similar occupancy distributions for the lower free energy states sampled by unbiased simulations, discarding a large size effect of our simulated system.

Each membrane system was equilibrated for $100 \mathrm{~ns}$ in the NPT ensemble at $1 \mathrm{~atm}$ and $303 \mathrm{~K}$. MD simulations for equilibration 
were performed using NAMD 2.933. A time step of 2 fs was used. Covalent bonds with hydrogen atoms of lipids were kept rigid using SHAKE ${ }^{34}$, and water molecules were kept rigid using SETTLE $^{35}$. The particle mesh Ewald method was employed to compute long-range electrostatic interactions ${ }^{36}$. The cutoff for Lennard-Jones interactions was set to $12 \AA$, with a switching distance of $10 \AA$. Pressure was controlled by the Langevin piston Nosé-Hoover method and the ratio of the unit cell in the $x-y$ plane was kept constant ${ }^{37}$. Temperature was controlled by the Langevin dynamics with a damping coefficient of $1 \mathrm{ps}^{-138}$.

\subsection{Collective Variables.}

For membranes in ionic solutions, there exists a competition between ion binding to water molecules and to certain binding sites of phospholipids ${ }^{9,20}$. Therefore, a bound state can be characterized by the ion's coordination number with lipid binding sites and its simultaneous coordination number with water molecules. A number of experiments ${ }^{3-6}$ and simulation works ${ }^{6-13}$ have indicated that metal cations bind directly to the oxygen atoms of the negative charged phosphate $\left(\mathrm{PO}_{4}^{-}\right)$and carbonyl $(\mathrm{C}=\mathrm{O})$ groups of lipid molecules. Accordingly, we defined two collective variables (CVs) to describe the ion's bound states: the coordination number between $\mathrm{Na}^{+}$ion and lipid (including cholesterol) oxygens (CLP), and the coordination number between $\mathrm{a} \mathrm{Na}^{+}$ion and water oxygens (CWT).

The coordination number ${ }^{39}$ is defined as

$$
s=\sum_{i \in G_{1}} \sum_{j \in G_{2}} s_{i j}
$$

where

$$
s_{i j}=\frac{1-\left(\frac{\left|\mathbf{r}_{i}-\mathbf{r}_{j}\right|-d_{0}}{r_{0}}\right)^{6}}{1-\left(\frac{\left|\mathbf{r}_{i}-\mathbf{r}_{j}\right|-d_{0}}{r_{0}}\right)^{12}} .
$$

For CLP, $G_{1}$ is the $\mathrm{Na}^{+}$ion, $G_{2}$ is all the lipid oxygens (including oxygen atom in cholesterol for cholesterol-containing membranes), $d_{0}=2.3 \AA$, and $r_{0}=0.25 \AA$. For CWT, $G_{1}$ is the $\mathrm{Na}^{+}$ ion, $G_{2}$ is all the water oxygens, $d_{0}=2.35 \AA$, and $r_{0}=0.25$ $\AA$. The values of $d_{0}$ and $r_{0}$ were determined from the radial distribution function $g(r)$, which was calculated from unbiased MD simulations on membrane systems contained 128 DMPC lipids, 6400 water molecules, and 46 pairs of $\mathrm{Na}^{+}$and $\mathrm{Cl}^{-}$ions (corresponding to the ionic concentration of $0.4 \mathrm{M}$ ). $d_{0}$ is the position of the first peak of $\mathrm{g}(\mathrm{r})$, and $r_{0}$ is the width at half maximum of the peak. The determination of the parameters for coordination number, i.e., $d_{0}$ and $r_{0}$, is essential for the accuracy of the resulting free energy surfaces. More details on the determination of $d_{0}$ and $r_{0}$ can be referred to our previous work ${ }^{20}$.

\subsection{Well-tempered Metadynamics Simulations.}

To obtain binding free energy landscapes of $\mathrm{Na}^{+}$at DMPCcholesterol membranes, we applied well-tempered metadynamics simulations ${ }^{40}$, a variant of metadynamics 41,42 capable of enhancing the sampling of coordination numbers in multiple $\mathrm{CV}$ dimensions. Two-dimensional (2D) well-tempered metadynamics simulations on six membrane systems with different choles- terol contents were performed based on the previously defined CVs using NAMD $2.9^{33}$ together with PLUMED2 plugin ${ }^{43}$ and the CHARMM36 force field ${ }^{27,28}$. After equilibration for $100 \mathrm{~ns}$ in the NPT ensemble as described above, $1000 \mathrm{~ns}$ well-tempered metadynamics simulations were performed on each system in the NVT ensemble at the temperature of 303K. The Gaussian widths for both CLP and CWT were set to 0.2. The initial Gaussian deposition rate was $0.3 \mathrm{kcal} / \mathrm{mol}$ per ps, with a bias factor of 5 . Despite the NPT ensemble is preferred in simulations of phospholipid membranes, we employed the NVT ensemble in applying well-tempered metadynamics due to technical reasons ${ }^{43}$. However, the 100ns NPT equilibration run brings the membrane to its equilibrium area per lipid (see Figure S1 in the Supplementary Information), which is then used in the production run to minimize the possible artifacts caused by the application of the NVT ensemble.

\section{Results and discussion}

The resulting 2D free energy surfaces (FES) of $\mathrm{Na}^{+}$bound to DMPC-cholesterol membranes with cholesterol concentrations $[\mathrm{CHOL}]=0-50 \%$ are shown in Figure $1 . \mathrm{Na}^{+}$is considered bound to the membrane for CLP $>0$ and unbound for CLP $=0$. There are a number of bound states (CLP $>0$ ) and several unbound states $(\mathrm{CLP}=0)$ in each FES, and each state can be indexed by (CWT, CLP). A common feature for all the [CHOL] cases is the global minimum of the FES located at the $(C W T=5, C L P=0)$ state, revealing that the hydration with 5 water molecules in the aqueous solution is most favorable for $\mathrm{Na}^{+}$. In addition, we observe a staircase pattern in all of the FES, which is the consequence of the ionic binding competition between water and lipids. As cholesterol concentration increases, the stable bound states (represented by red color in the FES), which are $\sim 1-4 \mathrm{kcal} / \mathrm{mol}$ higher than the global minimum, shift from the region with $\mathrm{CLP} \in[1,5]$ and $\mathrm{CWT} \in[0,5]$ for $[\mathrm{CHOL}]=0-10 \%$ to the region with $\mathrm{CLP} \in[1,4]$ and $\mathrm{CWT} \in[1,5]$ for $[\mathrm{CHOL}]=20-30 \%$, and further to the region with $\mathrm{CLP} \in[1,3]$ and $\mathrm{CWT} \in[2,5]$ for $[\mathrm{CHOL}]=40-50 \%$. This visible shifts of stable bound states clearly reveal that the binding competitiveness of lipid head groups has been diminished by cholesterol contents, and that the affinity of $\mathrm{Na}^{+}$to DMPC membranes becomes less favorable as cholesterol concentration increases, which is in agreement with experiments ${ }^{19}$. Although our results are strictly valid only at infinite ion dilution, a similar behavior should be expected at moderate ion concentrations, where ion-ion correlations are not important ${ }^{44}$.

A more quantitative representation of the above results is given in Figure 2, where the relative free energy $\Delta F$ as a function of CWT for several integer lipid binding levels (CLP) is extracted from the FES of Figure 1. Here we only represent the two extreme cases of $[\mathrm{CHOL}]=0 \%$ (continuous lines) and $[\mathrm{CHOL}]=$ $50 \%$ (dashed lines). For CLP=0, the hydration free energies of $\mathrm{Na}^{+}$unbound to the membrane are exactly the same for both [CHOL] cases, indicating that, as expected, the hydration of $\mathrm{Na}^{+}$ in the aqueous solution is not affected by the content of the membrane. There are several hydration free energy basins, and the most stable one is coordinated with five water molecules (which we establish as the reference state), in agreement with the $\mathrm{Na}^{+}$ 


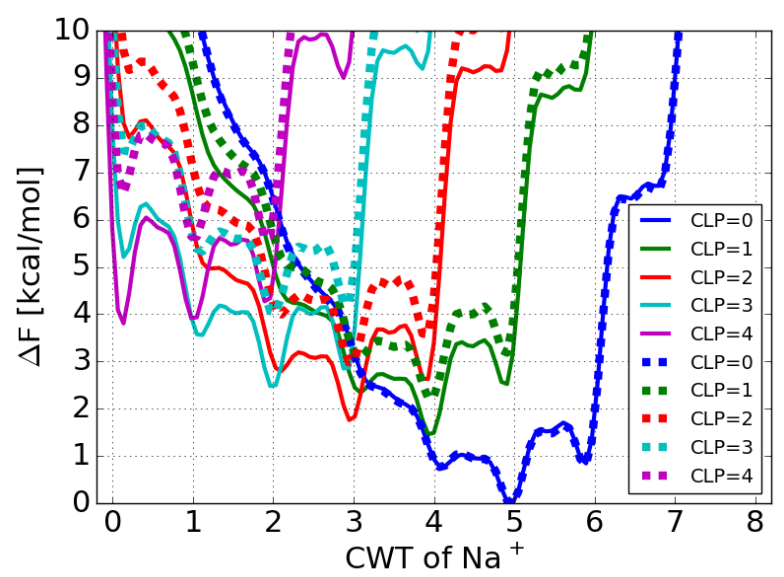

Fig. 2 Relative free energy $\Delta \mathrm{F}$ as a function of CWT at CLP $=0-4$ for $\mathrm{Na}^{+}$bound to DMPC membranes with cholesterol concentration [CHOL] of $0 \%$ (continuous lines) and $50 \%$ (dashed lines). The data are extracted from Figure 1.

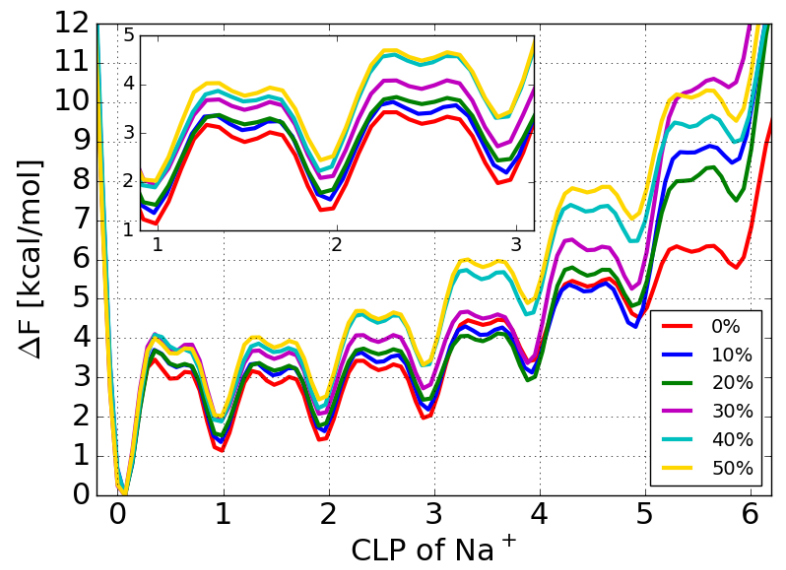

Fig. 3 Relative free energy $\Delta \mathrm{F}$ as a function of CLP for $\mathrm{Na}^{+}$bound to membranes with cholesterol concentration [CHOL] varying between $0 \%$ and $50 \%$. (Inset) Zoom in the low CLP region.

hydration number measured by experiments 45,46 . When bound to the membrane (CLP $>0$ ), the curves for the two cases become different. For each given CLP, the free energy profiles for [CHOL] $=50 \%$ case increase $1 \sim 2 \mathrm{kcal} / \mathrm{mol}$ compared to the cholesterolfree case $([\mathrm{CHOL}]=0 \%)$. Such increase evidences the decrease of $\mathrm{Na}^{+}$affinity to the membrane with high cholesterol content. However, for both [CHOL] cases, the corresponding free energy minima for CLP $=1,2,3,4$, are located at CWT $=4,3,2,1$, respectively, keeping 5 the total coordination number, which is the same as experimental hydration number of $\mathrm{Na}^{+}$in aqueous solution 45,46 .

In Figure 3, we represent the dependence of the relative binding free energy $\Delta F$ on CLP for membranes with various cholesterol concentrations after integrating out CWT according to

$$
\Delta F\left(s_{1}\right)=-k_{\mathrm{B}} T \log \int \exp ^{-\frac{\Delta F\left(s_{1}, s_{2}\right)}{k_{\mathrm{B}} T}} d s_{2}
$$

where $s_{1}$ and $s_{2}$ are CVs, $k_{\mathrm{B}}$ is the Boltzmann constant, and $\mathrm{T}$ is

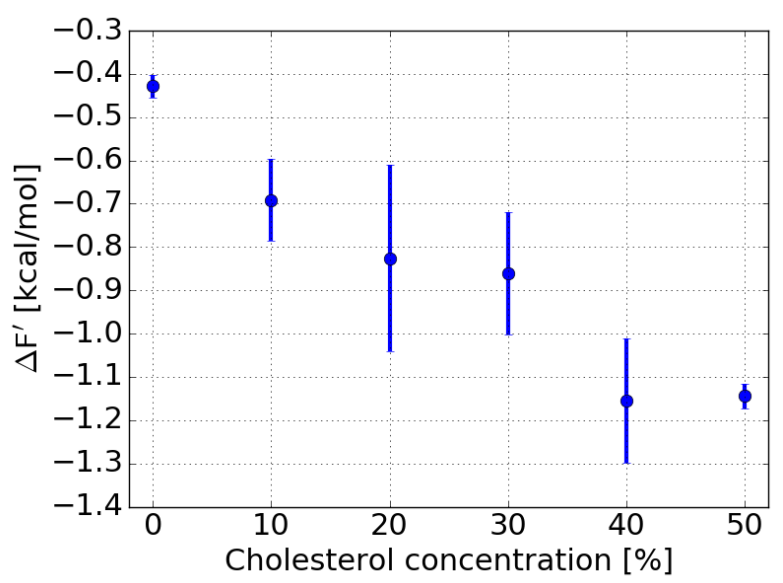

Fig. 4 Average values and standard deviations of $\Delta \mathrm{F}^{\prime}$, the free energy difference between unbound states $(C L P<0.5)$ and bound states $(C L P$ $>0.5)$ for different cholesterol concentrations.

the absolute temperature. As cholesterol concentration increases, the free energy profiles corresponding to bound states (CLP>0) raise monotonically. To understand these changes, we should remember the well-known condensing effect of cholesterol on lipid bilayers, which produces higher membrane rigidity and ordering ${ }^{47}$. For $[\mathrm{CHOL}] \leq 20 \%$, there are overlaps of the free energy profiles at low binding (CLP $\leq 3$ ), indicating that $\mathrm{Na}^{+}$is easily bound to the membranes with low cholesterol concentration. When $[\mathrm{CHOL}] \geq 30 \%$, the ionic binding is significantly reduced (free energy increases). Such transition in the ionic binding behavior at $[\mathrm{CHOL}] \approx 30 \%$ coincides with the phase transition point of DMPC-cholesterol membranes, in which membranes change from a liquid-disordered phase to a liquid-ordered phase ${ }^{17}$.

We monitor the convergence of the FES by calculating $\Delta \mathrm{F}$ ', the free energy difference between unbound states (CLP $<0.5$ ) and bound states (CLP $>0.5$ ) from the free energy profiles of CLP as the simulations proceed (see Figure S8 in the Supplementary Information). The average values and standard deviations of $\Delta F^{\prime}$ calculated from the last $100 \mathrm{~ns}$ of well-tempered metadynamics simulations are shown in Figure 4. The monotonic trend indicates that the energy gap between unbound and bound states increases with the increasing cholesterol concentration, which reduces the affinity of $\mathrm{Na}^{+}$to cholesterol-containing membranes. This is in good agreement with the results obtained from the previous Figures 1,2 , and 3 .

The above 2D FES (Figure 1 ) and $1 \mathrm{D}$ free energy $\Delta F$ as a function of CLP (Figure 3) are based on the consideration that all the oxygen atoms from DMPC lipids (i.e. phosphate and carbonyl groups) and cholesterol (hydroxyl group in the polar head) are equivalent binding sites for $\mathrm{Na}^{+}$. In order to understand the contributions of different head groups separately, we calculate the relative free energy $\Delta F$ as a function of CLP between $\mathrm{Na}^{+}$and oxygen atoms from different head groups by applying a reweighting technique ${ }^{48}$. As shown in Figure 5, for a given CLP, the binding free energy follows the order of $\mathrm{C}=\mathrm{O}<\mathrm{PO}_{4}^{-}$ $<-\mathrm{OH}$. Therefore, $\mathrm{C}=\mathrm{O}$ is the most favorable binding site for $\mathrm{Na}^{+}$, followed by $\mathrm{PO}_{4}^{-}$and then the $-\mathrm{OH}$ group of cholesterol. For 



Fig. 5 Relative free energy $\Delta \mathrm{F}$ as a function of CLP between $\mathrm{Na}^{+}$and oxygen atoms from different lipid head groups with cholesterol concentration [CHOL] varying between $0 \%$ and $50 \%$. The three different lipid head groups are: DMPC's phosphate group $\mathrm{PO}_{4}^{-}$(top figure); DMPC's carbonyl group $\mathrm{C}=\mathrm{O}$ (middle figure); and cholesterol's hydroxyl group $-\mathrm{OH}$ (bottom figure).

DMPC binding sites $\left(\mathrm{C}=\mathrm{O}\right.$ and $\left.\mathrm{PO}_{4}^{-}\right)$, we observe higher binding free energy basins and higher binding free energy barriers for higher cholesterol concentration, which is in accordance with above results obtained when different CLP are considered equivalent. The situation for the CLP between $\mathrm{Na}^{+}$and cholesterol is radically different. At low cholesterol concentration (10\%), only one cholesterol oxygen can be attached to $\mathrm{Na}^{+}$. For [CHOL] $\geq 20 \%$, it would be possible for $\mathrm{Na}^{+}$to bind up to two cholesterol oxygens. However, the free energy barriers for high cholesterol concentrations $(40-50 \%)$ are lower than those for the medium ones $(20-30 \%)$, which is in contrast to the trend observed for DMPC binding sites where higher free energy barrier corresponds to higher cholesterol concentration. It should be mentioned that the results reported in Figure 5 are influenced by the use of the new CHARMM36 force field, which uses specific parameters to describe the interaction of $\mathrm{Na}^{+}$with selected groups in the phospholipid (NBFIX) ${ }^{30}$.

Due to the high computational demands of the 2D welltempered metadynamics simulations, we used a small membrane, containing $72 \mathrm{DMPC} /$ cholesterol molecules. Although our tests using unbiased simulations of systems with 72 and 128 DMPC/cholesterol molecules suggest that large size effects can be discarded in describing ion binding to the membrane, a possible contribution from the finite size of the system cannot be completely ignored. The effect of the limited size of the system can also be relevant in regards to the heterogeneity of the membrane, which is important to ensure a proper sampling of the DMPC/cholesterol configurations of the membrane. We have run long enough simulations to allow complete mixing of lipids, but there might be size effects on the mixing of DMPC and cholesterol molecules which our approach was unable to evaluate.

\section{Conclusions}

We have performed systematic free energy calculations of $\mathrm{Na}^{+}$ bound to DMPC-cholesterol membranes by means of welltempered metadynamics simulations with two collective variables being the ion's coordination number to lipids (CLP) and to water (CWT). The free energy surfaces reveal the competition between binding of ion to water and to lipids. However, the binding competitiveness of lipid head groups is diminished by cholesterol contents. As cholesterol concentration increases, the ionic affinity to the membrane decreases, which is in agreement with experiments ${ }^{19}$. When cholesterol concentration [CHOL] $\geq 30 \%$, the ionic binding is significantly reduced. Such transition in the ionic binding behavior at $[\mathrm{CHOL}] \approx 30 \%$ coincides with the phase transition point of DMPC-cholesterol membranes, in which membranes change from a liquid-disordered phase to a liquidordered phase. In contrast, the hydration free energies of $\mathrm{Na}^{+}$ in aqueous solution are not affected by the cholesterol content of membranes. The most stable hydration for $\mathrm{Na}^{+}$with five water molecules is in good agreement with experiments. We have also evaluated the contributions of different lipid head groups to the binding free energy separately. The DMPC's carbonyl group $(\mathrm{C}=\mathrm{O})$ is the most favorable binding site for $\mathrm{Na}^{+}$, followed by DMPC's phosphate group $\left(\mathrm{PO}_{4}^{-}\right)$and then the hydroxyl group ($\mathrm{OH}$ ) of cholesterol.

The method employed can be widely applied to explore the free energy landscapes of ions at complex biological interfaces. Furthermore, provided the importance in a variety of biological processes of the interaction of ions and charged interfaces in aqueous solution, our approach could be extended to explore other problems in colloidal chemistry and biology, and could be helpful to deepen our understanding of specific ion effects on soft matter and biological systems.

\section{Acknowledgement}

The authors acknowledge computer resources and assistance provided by the Red Española de Supercomputación, through the Centre de Supercomputació de Catalunya. J.Y. acknowledges support from a FI-AGAUR fellowship of the Generalitat de Catalunya. J.M. gratefully acknowledges financial support from the Direcció Gen- 
eral de Recerca de la Generalitat de Catalunya (Grant 2009-SGR1003) and the Spanish MINECO for grant FIS2012-39443-C0201. C.C. acknowledges support from the Beatriu de Pinós program (BP-DGR 2011).

\section{References}

1 M. L. Berkowitz, D. L. Bostick and S. Pandit, Chem. Rev., 2006, 106, 1527-1539.

2 M. L. Berkowitz and R. Vácha, Acc. Chem. Res., 2012, 45, 7482.

3 H. Binder and O. Zschörnig, Chem. Phys. Lipids, 2002, 115, 39-61.

4 L. Herbette, C. A. Napolitano and R. V. McDaniel, Biophys. J., 1984, 46, 677-685.

5 N. N. Casillas-Ituarte, X. Chen, H. Castada and H. C. Allen, J. Phys. Chem. B, 2010, 114, 9485-9495.

6 P. Jurkiewicz, L. Cwiklik, A. Vojtíšková, P. Jungwirth and M. Hof, Biochim. Biophys. Acta, 2012, 1818, 609-616.

7 S. A. Pandit, D. Bostick and M. L. Berkowitz, Biophys. J., 2003, 84, 3743-3750.

8 R. A. Böckmann, A. Hac, T. Heimburg and H. Grubmüller, Biophys. J., 2003, 85, 1647-1655.

9 R. A. Böckmann and H. Grubmüller, Angew. Chem., Int. Ed., 2004, 43, 1021-1024.

10 A. Cordomí, O. Edholm and J. J. Perez, J. Phys. Chem. B, 2008, 112, 1397-1408.

11 A. A. Gurtovenko, J. Chem. Phys., 2005, 122, 244902.

12 A. A. Gurtovenko and I. Vattulainen, J. Phys. Chem. B, 2008, 112, 1953-1962.

13 R. Vácha, S. W. I. Siu, M. Petrov, R. A. Böckmann, J. BaruchaKraszewska, P. Jurkiewicz, M. Hof, M. L. Berkowitz and P. Jungwirth, J. Phys. Chem. A, 2009, 113, 7235-7243.

14 M. R. Vist and J. H. Davis, Biochemistry, 1990, 29, 451-464.

15 M. L. Berkowitz, Biochim. Biophys. Acta, 2009, 1788, 86-96.

16 F. J.-M. de Meyer, A. Benjamini, J. M. Rodgers, Y. Misteli and B. Smit, J. Phys. Chem. B, 2010, 114, 10451-10461.

17 C. L. Armstrong, M. A. Barrett, A. Hiess, T. Salditt, J. Katsaras, A.-C. Shi and M. C. Rheinstädter, Eur. Biophys. J., 2012, 41, 901-913.

18 X. L. R. Iraolagoitia and M. F. Martini, Colloids Surf., B, 2010, 76, 215-220.

19 A. Magarkar, V. Dhawan, P. Kallinteri, T. Viitala, M. Elmowafy, T. Róg and A. Bunker, Sci. Rep., 2014, 4, 5005.

20 J. Yang, C. Calero, M. Bonomi and J. Martí, J. Chem. Theory Comput., 2015, 11, 4495-4499.

21 S. Jo, T. Kim, V. G. Iyer and W. Im, J. Comput. Chem., 2008, 29, 1859-1865.

22 S. Jo, J. B. Lim, J. B. Klauda and W. Im, Biophys. J., 2009, 97, 50-58.

23 M. Pasenkiewicz-Gierula, Y. Takaoka, H. Miyagawa, K. Kitamura and A. Kusumi, J. Phys. Chem. A, 1997, 101, 36773691.

24 J. Yang, C. Calero and J. Martí, J. Chem. Phys., 2014, 140, 104901.
25 W. L. Jorgensen, J. Chandrasekhar, J. D. Madura, R. W. Impey and M. L. Klein, J. Chem. Phys., 1983, 79, 926-935.

26 J. A. D. MacKerell, D. Bashford, M. Bellott, J. R. L. Dunbrack, J. D. Evanseck, M. J. Field, S. Fischer, J. Gao, H. Guo, S. Ha, D. Joseph-McCarthy, L. Kuchnir, K. Kuczera, F. T. K. Lau, C. Mattos, S. Michnick, T. Ngo, D. T. Nguyen, B. Prodhom, W. E. Reiher, B. Roux, M. Schlenkrich, J. C. Smith, R. Stote, J. Straub, M. Watanabe, J. Wirkiewicz-Kuczera, D. Yin and M. Karplus, J. Phys. Chem. B, 1998, 102, 3586-3616.

27 J. B. Klauda, R. M. Venable, J. A. Freites, J. W. O'Connor, D. J. Tobias, C. Mondragon-Ramirez, I. Vorobyov, A. D. MacKerell and R. W. Pastor, J. Phys. Chem. B, 2010, 114, 7830-7843.

28 J. B. Lim, B. Rogaski and J. B. Klauda, J. Phys. Chem. B, 2012, 116, 203-210.

29 C. Valley, J. Perlmutter, A. Braun and J. Sachs, J. Membrane Biol., 2011, 244, 35-42.

30 R. M. Venable, Y. Luo, K. Gawrisch, B. Roux and R. W. Pastor, J. Phys. Chem. B, 2013, 117, 10183-10192.

31 M. Fyta and R. R. Netz, J. Chem. Phys., 2012, 136, 124103.

32 S. J. Marrink, and A. E. Mark, J. Phys. Chem. B, 2001, 105, 6122-6127.

33 J. C. Phillips, R. Braun, W. Wang, J. Gumbart, E. Tajkhorshid, E. Villa, C. Chipot, R. D. Skeel, L. Kalé and K. Schulten, J. Comput. Chem., 2005, 26, 1781-1802.

34 J.-P. Ryckaert, G. Ciccotti and H. J. Berendsen, J. Comput. Phys., 1977, 23, 327-341.

35 S. Miyamoto and P. A. Kollman, J. Comput. Chem., 1992, 13, 952-962.

36 U. Essmann, L. Perera, M. L. Berkowitz, T. Darden, H. Lee and L. G. Pedersen, J. Chem. Phys., 1995, 103, 8577.

37 W. Hoover, Phys. Rev. A, 1985, 31, 1695-1697.

38 S. E. Feller, Y. Zhang, R. W. Pastor and B. R. Brooks, J. Chem. Phys., 1995, 103, 4613.

39 M. Bonomi, D. Branduardi, G. Bussi, C. Camilloni, D. Provasi, P. Raiteri, D. Donadio, F. Marinelli, F. Pietrucci, R. A. Broglia and M. Parrinello, Comput. Phys. Commun., 2009, 180, 19611972.

40 A. Barducci, G. Bussi and M. Parrinello, Phys. Rev. Lett., 2008, 100, 020603.

41 A. Laio and M. Parrinello, Proc. Natl. Acad. Sci. USA., 2002, 99, 12562-12566.

42 A. Barducci, M. Bonomi and M. Parrinello, Wiley Interdiscip. Rev. Comput. Mol. Sci., 2011, 1, 826-843.

43 G. A. Tribello, M. Bonomi, D. Branduardi, C. Camilloni and G. Bussi, Comput. Phys. Commun., 2014, 185, 604-613.

44 A. Y. Grosberg, T. T. Nguyen and B. I. Shklovskii, Rev. Mod. Phys., 2002, 74, 329-345.

45 R. Mancinelli, A. Botti, F. Bruni, M. a. Ricci and a. K. Soper, J. Phys. Chem. B, 2007, 111, 13570-13577.

46 S. Varma and S. B. Rempe, Biophys. Chem., 2006, 124, 192199.

47 W.-C. Hung, M.-T. Lee, F.-Y. Chen and H. W. Huang, Biophys. J., 2007, 92, 3960-3967.

48 M. Bonomi, A. Barducci and M. Parrinello, J. Comput. Chem., 
2009, 30, 1615-1621. 